\title{
Xchange: Desenvolvimento de um protótipo para Mobile usando a Metodologia Design Sprint 2.0 Adaptada à Sala de Aula
}

\author{
Marcia Prante \\ Carla \\ Isabela \\ Asmann \\ Tubin \\ Bianchi \\ Marcia H.F.R. \\ Ana Carolina \\ Nardes \\ B. De Marchi
}

Programa de Pós-Graduação em Computação Aplicada (PPGCA) - Universidade de Passo Fundo (UPF)

Caixa Postal 611 - CEP 99001-970 - Passo Fundo - RS - Brasil

$\{180201,180196,128296,180200$, carolina $\} @$ upf.br

\section{RESUMO}

As metodologias ágeis são abordagens utilizadas no desenvolvimento de produtos de forma mais rápida e flexível; e estão sendo cada vez mais adotadas por empresas. A adoção de metodologias ágeis também vem se mostrando eficiente em sala de aula, modernizando a execução de projetos didáticos e trazendo novas experiências aos alunos e professores. Este trabalho tem como objetivo apresentar a prototipação de um software com o uso do método Design Sprint 2.0 e do framework Google Heart adaptados ao contexto de sala de aula. Os resultados obtidos demonstram que a metodologia pode ser uma ferramenta eficiente no contexto de sala de aula.

\section{PALAVRAS-CHAVE}

Aplicativo, Mobile, Design Sprint 2.0, Google Heart

\section{INTRODUÇÃO}

A popularidade dos smartphones e aplicativos móveis (apps) têm aumentado nos últimos anos [5]. Muitos problemas da vida moderna são solucionados com a utilização de aplicativos móveis. Os apps podem ser projetados com diferentes metodologias, entre elas o Design Sprint 2.0.

No Design Sprint 2.0 o processo de desenvolvimento ocorre em quatro dias, e envolve toda a equipe do projeto apenas nos dois primeiros dias [4]. No Design Sprint 1.0 eram necessários cinco dias em que todos os integrantes deveriam participar. Apesar das condições mais limitadas de sala de aula, estudo prévio demonstrou que a utilização da versão 1.0 se mostrou produtiva [7].

Neste trabalho, o objetivo foi avaliar o uso do Design Sprint 2.0, associado com o Google HEART framework, em sala de aula. Para tanto, foi prototipado o Xchange, um app que visa solucionar problemas de estoque parado em lojas de confecções de pequeno porte.

Permission to reproduce or distribute, in whole or in part, material extracted from this work, verbatim, adapted or remixed, as well as the creation or production from the content of such work, is granted without fee for noncommercial use, provided that the original work is properly credited.

IHC 2019 - TRILHA PÔSTERES E DEMONSTRAÇÕES, Outubro 21-25, 2019,

Vitória, Brasil. In Anais Estendidos do XVIII Simpósio Brasileiro sobre Fatores Humanos em Sistemas Computacionais. Porto Alegre: SBC.

(C) 2019 by the author(s), in accordance with the terms of the Creative Commons Attribution-NonCommercial 4.0 International Public License (CC BY-NC 4.0).

\section{O DESENVOLVIMENTO DO ESTUDO}

O estudo foi realizado por um grupo com quatro pessoas durante a disciplina de Projeto de Interfaces do Programa de Pós-Graduação em Computação Aplicada da Universidade de Passo Fundo. O processo levou quatro aulas com duração de 100 minutos de cada.

O objetivo foi desenvolver as principais telas do Xchange. Para isso, foi elaborada uma solução que permite que os responsáveis pelo controle do estoque dos estabelecimentos ofereçam e visualizem produtos para troca.

\section{Dia 1 - Mapear e Desenhar}

No primeiro dia do Design Sprint 2.0 foi realizado um mapeamento, com esboços das principais telas do produto.

A partir do mapeamento do produto a ser prototipado, foram entrevistados donos de estabelecimentos com vistas a validar a aceitação da solução. Com a ideia clara, utilizou-se o Google HEART Framework. O framework HEART foi criado para avaliar a qualidade da experiência do usuário (UX) [6].

Cada integrante fez anotações sobre o funcionamento do protótipo. Após, a equipe se reuniu e desenhou o mapa mental, que permitiu definir o escopo do projeto. Cada componente da equipe desenhou algumas telas, para que depois, o grupo pudesse votar nas melhores soluções.

\section{Dia 2 - Decisões e StoryBoard}

No dia 2 toda a equipe definiu o que seria prototipado, a partir de duas etapas:

i) Decidir: iniciou-se com o Heatmap, onde os membros da equipe colocaram seus votos nas partes dos esboços mais inspiradores. Foram dedicados 10 minutos para o Super Voto, que consiste na escolha das telas para prototipação.

ii) Storyboard: no Design Sprint 2.0 o processo de storyboard se tornou mais fácil em relação a versão 1.0, devido ao chamado User Test Flow. Trata-se de uma forma de exercício de avaliação e votação onde todos projetam seu próprio storyboard e depois são escolhidos um ou dois para a criação do protótipo [4]. A equipe realizou o User Test Flow individual com 06 posts com ações que indicavam como o usuário deveria percorrer $o$ protótipo. Na sequência, a equipe votou em um storyboard que seria prototipado. Definidas as telas, realizou-se a criação do storyboard (Figura 1). 


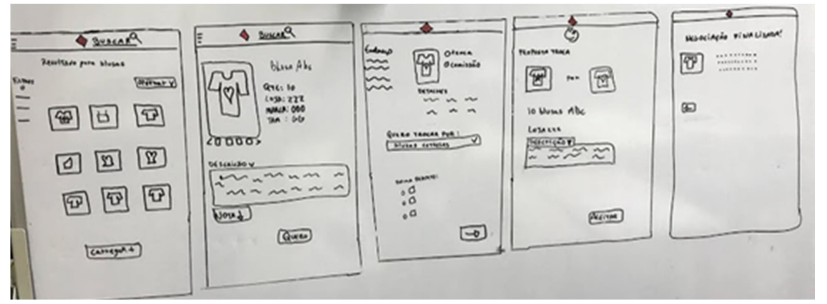

Figura 1: Storyboard para o protótipo

\section{Dia 3 - Prototipar}

Para a prototipação foi escolhida a ferramenta gratuita Quant-UX [1], que permite a criação de telas interativas com uma paleta de elementos, testes de usabilidade, opções para visualizar como o usuário interage com a aplicação, entre outras possibilidades.

As telas prototipadas foram a de pesquisa de produto, página de produto, carrinho, propor troca e negociações (Figura 2).

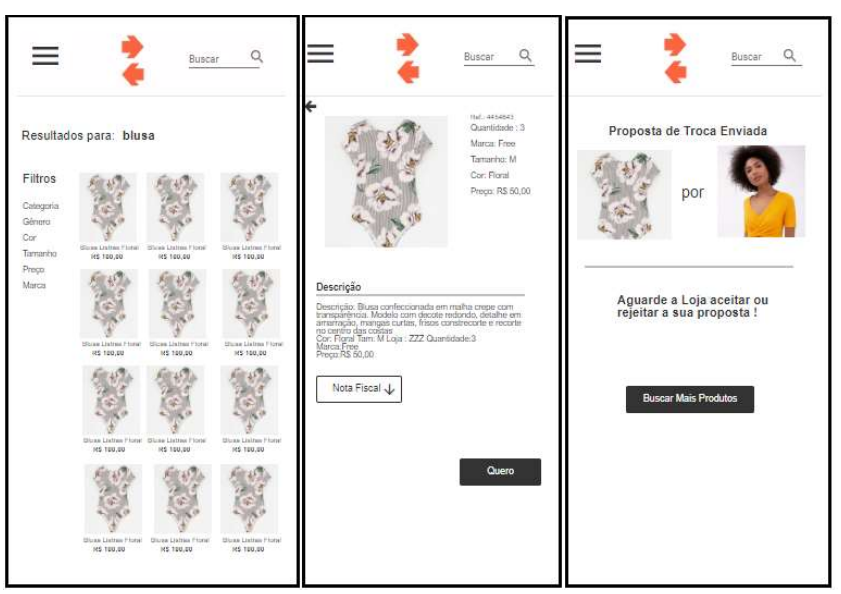

Figura 2: Principais telas do protótipo

\section{Dia 4 - Testar}

No Design Sprint 2.0 essa etapa é realizada de forma idêntica ao Design Sprint 1.0, a única diferença é o dia em que os testes ocorrem. Nesta etapa podem ser utilizadas algumas ferramentas de avaliação e de aceitação. A equipe optou por utilizar o System Usability Scale (SUS) [2]. É um método de escala numérica, onde são avaliados critérios como a efetividade do app e a satisfação dos usuários. O SUS é composto por um questionário com dez itens que permite fazer uma avaliação da usabilidade.

Os dados foram coletados por meio de formulário online via Google Docs, em um período de 07 dias. Além do questionário SUS, foram submetidas perguntas abertas, obtendo-se também dados qualitativos. Responderam a esta pesquisa 25 gerentes ou donos de loja. Também foram analisados dados do Quant-UX.

O Quant-UX contou com 84 interações, obtendo-se índices de satisfação de $20 \%$ a $100 \%$. Ao realizar o cálculo da média, alcançou-se o resultado de $72,1 \%$ de satisfação. Bangor et al. [3] concluíram que o SUS precisa ter um índice acima de $70 \%$. Considerando essa média, o aplicativo Xchange apresentou um bom grau de usabilidade, assim como no estudo de [7], que usou a versão 1.0. A Figura 3 apresenta o mapa de calor do Quant-UX, em que é possível observar as áreas mais clicadas nas telas.

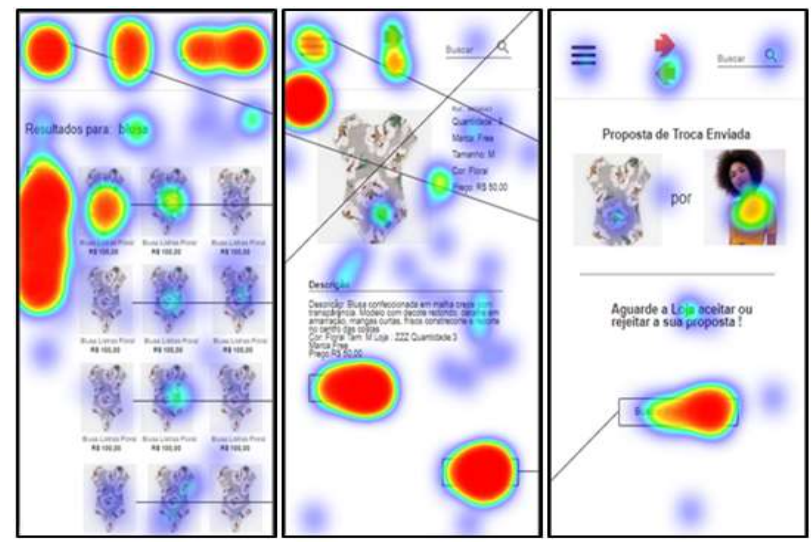

Figura 3: Mapa de calor das principais telas do protótipo

As respostas dos usuários foram satisfatórias, considerando que os usuários relataram facilidade no uso e que não mudariam nada no aplicativo. No entanto, alguns usuários relataram que não conseguiram navegar ou compreender a forma de busca. Tal fato pode ter ocorrido por se tratar de um protótipo, onde o produto é versão de um sistema que antecede à principal, geralmente reduzida, para ser aperfeiçoada [8].

\section{CONCLUSÃO}

O aplicativo prototipado alcançou bons resultados, de acordo com as avaliações realizadas. Desse modo, concluiu-se que a aplicação do Design Sprint 2.0, em conjunto com o Google HEART framework, mostrou-se uma metodologia viável para uso em sala de aula, mesmo sendo necessárias algumas adaptações.

\section{REFERENCIAS}

[1] QUANT-UX. Quant-UX: Prototype, Test and Learn. https://www.quant-ux.com/.

[2] Thomas Mansūr. Design Sprint 2.0: o que há de novo? https://brasil.uxdesign.cc/design-sprint-2-0-o-que-h\%C3\%A1-de-novod29fab15d782.

[3] BANGOR, A., KORTUM, P., e MILLER, J. Determining What Individual SUS Scores Mean: Adding an Adjective Rating Scale. Journal of Usability Studies, v.4, n.3, p.114-123, Maio 2009.

[4] Wesley Simões. Design Sprint 2.0: Como funciona? https://medium.com/ixdabh/design-sprint-2-0-b73e86c7cb9d.

[5] YU, Peng. Yeung, Ching-man Au. ACM, 2014. App Mining: Finding the Real Value of Mobile Applications Proceedings of the 23rd International Conference on World Wide Web.

[6] Emily Bonnie. How to Use the Google HEART Framework to Measure and Improve Your App's UX. https://clevertap.com/blog/google-heart-framework.

[7] Bellei, A., et al. 2017 "Projetando uma Interface de Jogo de Interação Gestual com a Metodologia Design Sprint Adaptada à Sala de Aula." XVI Simpósio Brasileiro

sobre Fatores Humanos em Sistemas Computacionais, IHC, Joinville. DOI: 10.13140/RG.2.2.30820.68487

[8] C. Bela and C. Palhais, 2015. "PROTOTIPAGEM de um produto,". https://repositorio.ul.pt/bitstream/10451/29163/2/ULFBA_TES_942.pdf. Conference Name:ACM Woodstock conference 